\title{
ON THE EVOLUTION OF PARTICLE TRANSITIONS FROM ONE WELL TO ANOTHER IN A DOUBLE-WELL POTENTIAL
}

\author{
V. S. Olkhovsky, S. P. Maidanyuk \\ Institute for Nuclear Research, National Academy of Sciences of Ukraine, \\ 47 Nauky Pr., Kyiv-22, UA-252650, Ukraine \\ (Received June 22, 1998; received in final form December 9, 1998)
}

\begin{abstract}
The Poincare's period of particle oscillations between wells is obtained in a double-well potential. The dependencies of the oscillation period on transmission coefficient and on distance between levels are obtained. The cases of squared potentials and some potentials having rounded off forms are considered specifically.
\end{abstract}

Key words: double-well potential, tunneling, oscillation period, transmission coefficient.

PACS numbers: 03.65.Bz; 03.65.Ge.

\section{INTRODUCTION}

The tunneling of a particle through the potential barrier is an essentially quantum phenomenon. This process involves propagation of a particle through a classically inaccessible region. The complete information about the tunneling of a particle from the solution of Schrödinger equation with appropriate boundary conditions can be obtained in all regions. But in practice, the exact solution of Schrödinger equation can be found for some simplest forms of potentials and it is difficult enough to obtain the exact solution for an arbitrary potential form. For this reason the approximation methods are used for finding solutions for potentials of a specific form. But exact solutions, which were found, are of great importance, because they allow to analyze the tunneling process in general.

It is sufficiently difficult to obtain solutions for multidimension potential forms. Therefore, in the present work only one-dimensional case is considered, for which exact solutions are obtained for some simple forms of potential having two wells separated by barrier. Every specific form of potential in comparison with the other ones requires the use of a specific approach for solving the problem, allowing some features of tunneling to be more pronounced, the others remaining in the shade. Having analyzed the use of boundary conditions for solving the problem, we proposed to divide various shapes of doublewell potentials into two classes: squared potentials and potentials having rounded off forms. Squared doublewell potentials have exact analytic solutions (which can be expressed through elementary functions). Rounded off double-well potentials have the solutions expressed through special functions (if these solutions exist). In the present work after qualitative analysis of energy levels of various forms of the double-well potential the problem of squared potential and the problem of rounded off potential (the Morse's potential) are considered separately in the following two sections.

The period of particle oscillations between two wells is one of the most important parameters which characterize the process of tunneling. We can obtain its value falling back on the energy levels of the system. For this at the beginning we will confine ourselves to class of systems, for which the distances between energy levels have the exactly determined largest common devizor $\Delta$ and the following condition is fulfilled:

$$
E_{n}=E_{0}+\Delta * l_{n}
$$

where $l_{n} \in 0, N$. In the general case within the region of discrete energy spectrum the states of these systems are described by the wave packet as follows [3]

$$
\begin{aligned}
\psi(t) & =\sum_{n} g_{n} \varphi_{n}(x) e^{-i\left(E_{n}-E_{0}\right) t / \hbar} \\
& =\sum_{n} g_{n} \varphi_{n}(x) e^{-i \Delta l_{n} t / \hbar}
\end{aligned}
$$

where $\varphi_{n}(x)$ is orthonormal wave functions of stationary states of the system satisfying the equation $\widehat{H} \varphi_{n}(x)=$ $E_{n} \varphi_{n}(x) ; \hat{H}$ is the Hamiltonian of the system; $\sum_{n}\left|g_{n}\right|^{2}=$ 1 and the insignificant factor $e^{-i E_{0} t / \hbar}$ is omitted which is common for all terms of the sum $\sum_{n}$. Let us select the moment $t=0$ as the origin of time reference.

Let the function $\psi(t)$ be determined on the region $\left[-\frac{\pi \hbar}{\Delta}, \frac{\pi \hbar}{\Delta}\right]$ and satisfy the Dirichlet's conditions [11]: (a) it can divide this region into a finite number of regions in which the function $\psi(t)$ will be continuous, monotonic and bounded; (b) if $t_{0}$ is the discontinuity point of the function $\psi(t)$, then $\psi\left(t_{0}+0\right)$ and $\psi\left(t_{0}-0\right)$ exist. In that case expression (1.2) is an expansion of the function $\psi(t)$ into the Fourier series with respect to $t$ which is converging in all the points of the region $\left[-\frac{\pi \hbar}{\Delta}, \frac{\pi \hbar}{\Delta}\right]$. Then the function $\psi(t)$ is periodic in time and period of the oscillations (the time of Poincare's cycle) is given by

$$
T=\frac{2 \pi \hbar}{\Delta}
$$


Expression (1.3) determines the period of oscillations of the wave packet, if energy levels in the region of a discrete spectrum have exactly determined common largest divizor $\Delta$ and condition (1.1) is fulfilled.

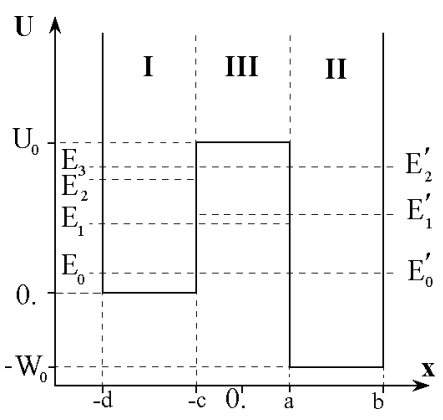

Fig. 1. Double-well squared potential.

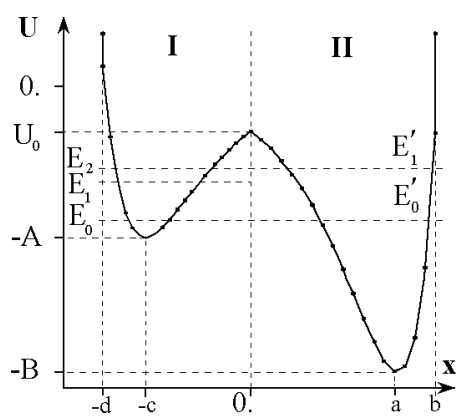

Fig. 2. Double-well rounded off potential.

In a general case for the systems for which the distances between energy levels in the region of discrete spectrum do not have the exactly determined divizor $\Delta$ one can select the 'quasi-cycles' with the given degree of accuracy, for which the state of the system approaches the maximum degree the initial state after a 'quasi-cycle' time [1]. The states of such systems are localized in a confined volume of space and the time of Poincare's cycle (which includes the required number of 'quasi-cycles') can be determined with given degree of accuracy. To find the information about some parameters for quantum systems evolving with time in the region of discrete spectrum see $[1,6,7]$.

Since the period of particle oscillations between wells is obtained on the basis of energy eigenvalues, much attention is paid to the problem of solving eigenvalue equations. For some forms of potential the transmission coefficient through the barrier is found. This parameter can be obtained using two approaches: in the region of continuous spectrum for the particle which is incident upon the barrier, with asymptotic velocity $\hbar k / m$, and, when the condition of WKB approximation is fulfilled, in the region of discrete spectrum for the particle which initially is located in one well and then is tunneling to another well. In case of double-well squared potential the comparison of these two approaches for calculating the transmission coefficient through the barrier is performed. For symmetric double-well potentials the dependence of transmission coefficient (which is found using one of the approaches) on the period of particle oscillations between wells is analyzed.

\section{ANALYSIS OF THE POSSIBILITY OF PARTICLE TUNNELING THROUGH A BARRIER}

The qualitative estimation - will the particle oscillate between wells or not - can be obtained on the basis of analysis of solutions of stationary Schrödinger equations which are found for every well.

Let us divide various forms of double-well potentials into two classes: squared potentials (see Fig. 1) and potentials, which have rounded off forms (see Fig. 2). To obtain the energy levels, the Schrödinger equation is solved separately in every region (3 regions for squared potential, see Fig. 1; 2 regions for potential having rounded off form, see Fig. 2).

As a result of this solution, the wave functions $\varphi_{i}(x)$ are found in every region, and these functions must be continuous and bounded (we consider discrete spectrum $E<U_{0}$ ) all over the region of its determination (here, $i$ is the number of region). If wave function is expressed through special or elementary functions, then it will be bounded everywhere in the region $-d<x<b$ (with the exception of some cases of hypergeometric functions which must be considered separately, see $[9,10])$, and in points $x=-d$ and $x=b$ the boundary conditions are is given by

$$
\varphi_{1}(-d)=0, \varphi_{2}(b)=0
$$

This conditions determine eigenvalues for energy levels as for discrete levels. Condition of continuity of wave function and its derivative in the region $-d<x<b$ requires the equality of solutions $\varphi(x)$ and $d \varphi(x) / d x$ for the adjacent regions in the points of boundary between these regions (in these points the discontinuity of derivative is possible).

At the beginning we consider squared potential (see Fig. 1). For particle located in left well (region 1) we analyze the possible cases of its propagation to right well in result of tunneling through the barrier. Among them we select the following cases:

1. In regions 1,2 and 3 there are equal levels of $E_{0}$ (see Fig. 1) which are found from Schrödinger equation for every region separately. Then the particle can propagate through the barrier along level $E_{0}$. (In this case the transitions between levels are not required for tunneling). We obtain the eigenvalue of energy level $E_{0}$ from system (2.1) and the following system:

$$
\varphi_{1}(-c)=\varphi_{3}(-c), \quad \varphi_{1}^{\prime}(-c)=\varphi_{3}^{\prime}(-c)
$$




\section{S. OLKHOVSKY, S. P. MAIDANYUK}

$$
\varphi_{3}(a)=\varphi_{2}(a), \quad \varphi_{3}^{\prime}(a)=\varphi_{2}^{\prime}(a) .
$$

2. In regions 1 and 3 there are equal levels of $E_{1}$, but such levels are absent from region 2 . In regions 2 and 3 there are equal levels of $E_{1}^{\prime}$, but such levels are absent from region 1 . Then the particle initially located on level $E_{1}$ in the left-side well, can not propagate to the rightside well along this level. But in region 3 wave functions corresponding to levels $E_{1}$ and $E_{1}^{\prime}$ are not equal to zero. Therefore, in this region the matrix element of transition from level $E_{1}$ to level $E_{1}^{\prime}$ (and on the contrary) is not equal to zero. Therefore, the particle which is initially located on level $E_{1}$ in the left-side well can propagate to the right-side well with a transition from level $E_{1}$ to level $E_{1}^{\prime}$. The transition $E_{1} \rightarrow E_{1}^{\prime}$ takes place in the region of barrier 3 . Eigenvalue of level $E_{1}$ can be obtained from the system:

$$
\begin{array}{ll}
\varphi_{1}(-c)=\varphi_{3}(-c), & \varphi_{1}^{\prime}(-c)=\varphi_{3}^{\prime}(-c), \\
\varphi_{1}(-d)=0, & \varphi_{3}(a)=0 .
\end{array}
$$

In this fashion, one can find the eigenvalue for level $E_{1}^{\prime}$ from the following system:

$$
\begin{array}{ll}
\varphi_{2}(a)=\varphi_{3}(a), \quad \varphi_{2}(b)=0, \\
\varphi_{2}^{\prime}(a)=\varphi_{3}^{\prime}(a), \quad \varphi_{3}(-c)=0 .
\end{array}
$$

3 . In region 1 the particle is located on level $E_{2}$ and this level is absent from regions 2 and 3 . Also in regions 1,2 and 3 there are equal levels of $E_{2}^{\prime}$. In this case the particle can not propagate along level $E_{2}$ to the right well and to region 3 of the barrier (there is a full reflection of the particle along level $E_{2}$ ). But the particle can make transition on level $E_{2} /$ in region 1 and then it can propagate to regions 2 and 3 along this level. One can find the eigenvalue of level $E_{2}$ from the following system:

$$
\varphi_{1}(-d)=0, \varphi_{1}(-c)=0
$$

The eigenvalue of level $E_{2}^{\prime}$ satisfies system (2.1) and system $(2.2)$.

More concretely the first case will be considered in one of the next sections. Note, that at the symmetry of potential $\left(d=b, c=a, W_{0}=0\right)$ for every level of left well one can find the appropriate level in right well and conversely. Therefore, for cases considered above only the first case is possible for this potential.

Now we consider the potential which has a rounded off form (see Fig. 2). Let the particle be localized in the left well. Among possible cases, in which the particle can propagate through a barrier to right well, we select the following cases:

1. In regions 1 and 2 there are equal levels $E_{0}$. Then the particle can tunnel from left well to right well along level $E_{0}$. The eigenvalue for this level can be found from system (2.1) and the following system:

$$
\varphi_{1}(0)=\varphi_{2}(0), \varphi_{1}^{\prime}(0)=\varphi_{2}^{\prime}(0)
$$

2. In left well the particle is located on level $E_{1}$ and this level is absent in region 2. But in regions 1 and 2 there are equal levels $E_{1}^{\prime}$. Then the particle cannot tunnel from the left well into the right one along level $E_{1}$. But at first it can make a transition from level $E_{1}$ to level $E_{1}^{\prime}$ in region 1 and then it can propagate to region 2 along level $E_{1}^{\prime}$. A system for finding the eigenvalue of level $E_{1}$ is given by

$$
\varphi_{1}(-d)=0, \varphi_{1}(0)=0
$$

The eigenvalue for level $E_{1}^{\prime}$ can be obtained from system (2.1) and system (2.6).

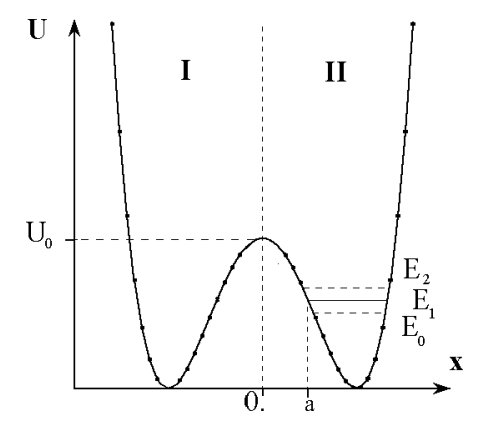

Fig. 3. Splitting of energy levels in symmetric potential.

In one of the next sections the problem with potential of such a form will be considered more concretely. As an example, the double-well Morse's potential is selected. Also note, that for potential of the rounded off form (as in case of the squared potential) only the first case is possible when the potential is symmetric.

\section{A DEPENDENCE OF THE DISTANCE BETWEEN THE TWO CLOSELY LOCATED LEVELS ON THE TRANSMISSION COEFFICIENT THROUGH A BARRIER IN A QUASI-CLASSIC SYMMETRIC POTENTIAL}

Consider potential $U(x)$ which has two symmetric wells separated by a barrier (see Fig. 3). If the barrier is not penetrable, then there are energy levels $E_{0}$ corresponding to oscillations of the particle only in one well. The possibility of particle transitions between the wells leads to splitting of every level $E_{0}$ into two closely located levels $E_{1}$ and $E_{2}$.

We consider the case, when potential $U(x)$ is quasiclassic. Then the splitting value $\Delta E$ can be obtained through the wave function $\varphi_{0}(x)$ which determined with accuracy to first order terms in $\hbar$, as follows [2] 


$$
\begin{aligned}
\Delta E & =E_{2}-E_{1}=\frac{2 \hbar^{2}}{m} \varphi_{0}(0) \varphi_{0}^{\prime}(0) \\
& =\frac{w \hbar}{\pi} \exp \left(-\frac{1}{\hbar} \int_{-a}^{a}|p| d x\right)
\end{aligned}
$$

where

$$
\begin{aligned}
\varphi_{0}(0) & =\sqrt{\frac{w}{2 \pi v_{0}}} \exp \left(-\frac{1}{\hbar} \int_{0}^{a}|p| d x\right), \\
\varphi_{0}^{\prime}(0) & =\frac{m v_{0}}{\hbar} \varphi_{0}(0)
\end{aligned}
$$

and $v_{0}=\sqrt{2\left(U_{0}-E\right) / m}, p$ is the system momentum, $w=2 \pi / T$ is frequency of classic periodic oscillations; $a$ is turning point corresponding to level $E_{0}$ (see Fig. 3).

Transmission coefficient $D$ through the barrier in the WKB approximation is determined by [2]

$$
D=\text { const } \cdot \exp \left(-\frac{2}{\hbar} \int_{-a}^{a}|p| d x\right)
$$

where proportionality factor const is determined by the accuracy of the WKB approximation and is equal to 1 with the accuracy of the first order terms in $\hbar$ (see [2]). Taking into consideration expressions (3.1) and (3.3) we can write

$$
\Delta E=\mathrm{const} \cdot \frac{w \hbar}{\pi} \sqrt{D}
$$

Here, transmission coefficient $D$ is determined by expression (3.3) for the discrete energy spectrum. In accordance with the conditions of using the WKB approximation expression (3.4) determining the dependence of level splitting $\Delta E$ on transmission coefficient $D$ is used only for small values of $D$.

Now we consider some cases, for which there are exact analytical solutions.

\section{DOUBLE-WELL INFINITE SQUARED POTENTIAL}

Consider a system the potential of which consists of two squared wells separated by squared barrier of finite height (see Fig. 1). This potential is given by

$$
U(x)=\left\{\begin{array}{rlr}
\infty, & \text { for } x<-d, x>b ; & \\
0, \text { for }-d<x<-c, & \text { (region I) } \\
U_{0}, & \text { for }-c<x<a, & \text { (region III) } \\
-W_{0}, & \text { for } a<x<b, & \text { (region II) }
\end{array}\right.
$$

In case of discrete energy spectrum in the region of $U_{0}>E>0$ we find the solution of the stationary Schrödinger equation in the form:

$$
\varphi(x)= \begin{cases}a_{1} \sin (k(x+d)), & \text { for }-d<x<-c \\ a_{2} e^{\chi x}+b_{2} e^{-\chi x}, & \text { for }-c<x<a \\ a_{3} \sin \left(k_{3}(x-b)\right), & \text { for } a<x<b\end{cases}
$$

Here, the following coefficients are used:

$$
\begin{aligned}
k & =\frac{1}{\hbar} \sqrt{2 m E}, \\
k_{3} & =\frac{1}{\hbar} \sqrt{2 m\left(E+W_{0}\right)} \\
\chi & =\frac{1}{\hbar} \sqrt{2 m\left(U_{0}-E\right)} .
\end{aligned}
$$

Let's consider the case of particle oscillations between wells along one energy level (without transitions between energy levels). Unknown coefficients and these energy levels can be found from continuity conditions of wave function in boundary points $x=-c, x=a$ and from the following normalization condition:

$$
\int_{-\infty}^{+\infty}|\varphi(x)|^{2} d x=1
$$

Ultimately, we obtain unknown coefficients:

$$
\begin{aligned}
a_{1} & =\left\{\frac{d-c}{2}+\frac{a+c}{2}\left[\sin ^{2}(k(d-c))-\frac{k^{2}}{\chi^{2}} \cos ^{2}(k(d-c))\right]\right. \\
& +\left(\frac{\sin (k(d-c))-k / \chi \cos (k(d-c))}{\sin \left(k_{3}(b-a)\right)+k_{3} / \chi \cos \left(k_{3}(b-a)\right)}\right)^{2} e^{-2 \chi(a+c)}\left[\frac{b-a}{2}+\frac{1}{4 k_{3}} \sin \left(2 k_{3}(a-b)\right)\right] \\
& -\frac{1}{4 k} \sin (2 k(d-c))+\frac{(\sin (k(d-c))+k / \chi \cos (k(d-c)))^{2}}{8 \chi}\left(e^{2 \chi(a+c)}-1\right)
\end{aligned}
$$




$$
\begin{gathered}
\left.+\frac{(\sin (k(d-c))-k / \chi \cos (k(d-c)))^{2}}{8 \chi}\left(1-e^{-2 \chi(a+c)}\right)\right\}^{-1 / 2} \\
a_{2}=a_{1} \frac{1}{2} e^{\chi c}(\sin (k(d-c))+k / \chi \cos (k(d-c))), \\
b_{2}=a_{1} \frac{1}{2} e^{-\chi c}(\sin (k(d-c))-k / \chi \cos (k(d-c))), \\
a_{3}=a_{1} e^{-\chi(a+c)} \frac{k / \chi \cos (k(d-c))-\sin (k(d-c))}{k_{3} / \chi \cos \left(k_{3}(b-a)\right)+\sin \left(k_{3}(b-a)\right)} .
\end{gathered}
$$

The eigenvalue equations for this potential are given by

$$
\begin{aligned}
& E=\hbar^{2} k^{2} / 2 m \\
& \frac{\sin \left(k_{3}(b-a)\right)-k_{3} / \chi \cos \left(k_{3}(b-a)\right)}{\sin \left(k_{3}(b-a)\right)+k_{3} / \chi \cos \left(k_{3}(b-a)\right)} \cdot \frac{\sin (k(d-c))-k / \chi \cos (k(d-c))}{\sin (k(d-c))+k / \chi \cos (k(d-c))}=e^{2 \chi(a+c)} .
\end{aligned}
$$

Now we consider the symmetric case of $\left(d=b, c=a, W_{0}=0\right)[2,4]$. The wave function became symmetric or antisymmetric:

$$
\varphi_{n}(x)= \begin{cases}a_{1} \sin \left(k_{n}(x+b)\right), & \text { for }-b<x<-a \\ b_{2}\left((-1)^{n} e^{\chi x}+e^{-x x}\right), & \text { for }-a<x<a \\ a_{1}(-1)^{n} \sin \left(k_{n}(b-x)\right), & \text { for } a<x<b .\end{cases}
$$

where $n \in 0, N$. The unknown coefficients $a_{1}$ and $b_{2}$, obtained from expressions (4.5) and (4.6), are given by

$$
\begin{aligned}
& a_{1}=\left\{b-a+\frac{(\sin (k(b-a))-k / \chi \cos (k(b-a)))^{2}}{4 e^{2 \chi a}}\left((-1)^{n} 2 a+\frac{e^{2 \chi a}-e^{-2 \chi a}}{\chi}\right)\right\}^{-1 / 2} \\
& b_{2}=a_{1} \frac{\sin (k(b-a))-k / \chi \cos (k(b-a))}{2 e^{\chi^{a}}} .
\end{aligned}
$$

The wave vector $k$ is transformed to form:

$$
k=\frac{1}{b-a}\left\{-\arcsin \left[\frac{1}{\sqrt{1+\frac{\chi^{2}}{k^{2}}\left(\frac{(-1)^{n}-e^{2 \chi a}}{(-1)^{n}+e^{2 \chi a}}\right)^{2}}}\right]+\pi n\right\} .
$$

Consider the particle propagating from left to right in potential (4.1) with the asymptotic velocity $\hbar k / m$ and energy $E<U_{0}$. For it the wave function can be written as follows

$$
\varphi(x)= \begin{cases}(\hbar k / m)^{-1 / 2} *\left(e^{i k x}-A e^{-i k x}\right), & \text { for } x<-c \\ (\hbar k / m)^{-1 / 2} *\left(B e^{\chi x}+B^{\prime} e^{-\chi x}\right), & \text { for }-c<x<a \\ (\hbar k / m)^{-1 / 2} * C e^{i k x}, & \text { for } x>a\end{cases}
$$

Coefficients $A, B, B^{\prime}$ and $C$ are obtained from continuity conditions for $\varphi(x)$ and $d \varphi(x) / d x$ at the points $x=-c$ and $x=a$. The transmission coefficient $D$ and reflection coefficient $R$ calculated as the ratio of the flux of incident wave in region III to the flux of transmitted wave in region I or reflected wave in region III, are given by

$$
D=\frac{4 k k_{3} \chi^{2}}{\left(k k_{3}+\chi^{2}\right)^{2} \sinh ^{2}(\chi(a+c))+\chi^{2}\left(k-k_{3}\right)^{2} c h^{2}(\chi(a+c))+4 k k_{3} \chi^{2}},
$$




$$
R=\frac{\left(k k_{3}+\chi^{2}\right)^{2} \sinh ^{2}(\chi(a+c))+\chi^{2}\left(k-k_{3}\right)^{2} c h^{2}(\chi(a+c))}{\left(k k_{3}+\chi^{2}\right)^{2} \sinh ^{2}(\chi(a+c))+\chi^{2}\left(k-k_{3}\right)^{2} c h^{2}(\chi(a+c))+4 k k_{3} \chi^{2}} .
$$

We find the values $D$ and $R$ for transmission of the particle through the barrier in continuous energy spectrum. But comparing the transmission coefficient $D$ with its small values determined by expression (4.11) for symmetric case with that determined by expression (3.3) which is obtained in the WKB approximation for a discrete energy spectrum shows that both the approaches give identical formulations with accuracy to the normalized constant: $D=$ const $\cdot \exp (-2 \chi a)$, where const is determined by the accuracy of the WKB approximation and is equal to 1 in terms of the first order in $\hbar[2,5]$. In this sense, we will formally consider expression (4.11) as the determination of transmission and reflection coefficients for the discrete energy spectrum. The values $k$ and $\chi$, used in expression (4.11), can be obtained from equation (4.7) or (4.10).
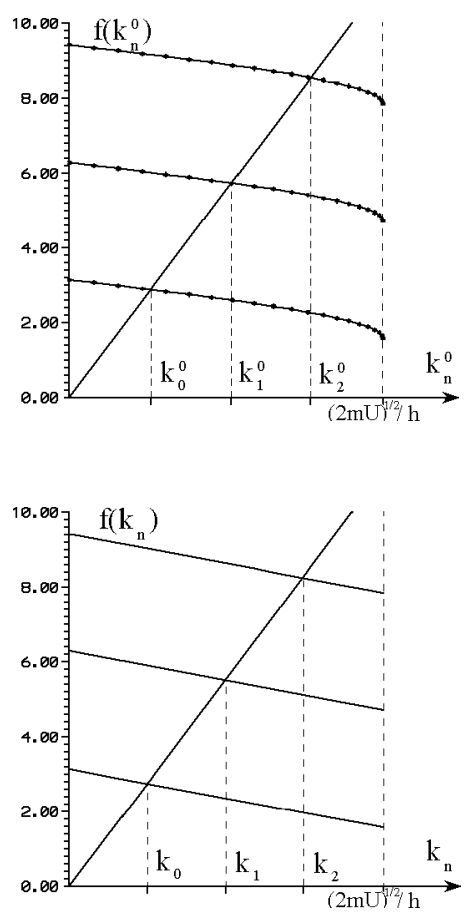

Fig. 4. The values $k_{n}^{0}$ are exact graphic solutions of set (4.15) and the values $k_{n}$ are graphic solutions on this set, where the second equation is changed to its linear approximation $f_{2}\left(k_{n}\right)$.

We analyze the periodicity of particle oscillations between wells in a symmetric potential. For this we consider equation (4.10) which can be written as

$$
\begin{aligned}
f_{1}\left(k_{n}\right) & =k_{n}(b-a), \\
f_{2}\left(k_{n}\right) & =-\arcsin \left\{\frac{1}{\sqrt{1+\frac{\chi^{2}}{k^{2}}\left(\frac{(-1)^{n}-e^{2 \times a}}{(-1)^{n}+e^{2 \times a}}\right)^{2}}}\right\} \\
& +\pi n .
\end{aligned}
$$

The graphic analysis of exact solutions of system (4.13) gives a number of values $k_{n}^{0}$ (see Fig. 4). Changing the second equation of set (4.13) to its linear relation $f_{2}\left(k_{n}\right)$, which can be obtained by linear approximation, we find the following values of $k_{n}$ :

$$
\begin{aligned}
k_{n} & =k_{0}(2 n+1), \\
E_{n} & =E_{0}(2 n+1)^{2}=E_{0}+4 E_{0} n(n+1) \\
& =E_{0}+\Delta l_{n}, \\
\Delta & =4 E_{0} .
\end{aligned}
$$

where $n, l_{n} \in 0, N$. From expressions (4.14) we obtain period $T$ of particle oscillations between the wells:

$$
\begin{aligned}
T & =\frac{2 \pi \hbar}{\Delta}=\frac{\pi \hbar}{2 E_{0}}=\frac{\pi \hbar}{2} \frac{(2 n+1)^{2}}{E_{n}} \\
& =\frac{\pi m}{\hbar k_{n}^{2}}(2 n+1)^{2} .
\end{aligned}
$$

For values $k_{n}$ the accuracy $\pi /(2(b-a))$ is used. The series of solutions $k_{n}$ is bounded by the maximum value $k_{N}$, where $N$ (the number of energy levels in the region $\left.E<U_{0}\right)$ satisfies the following condition:

$$
0<N<\frac{\sqrt{2 m U_{0}}-\hbar k_{0}}{2 \hbar k_{0}} .
$$

From expression (4.15) we obtain:

$$
k_{n}^{2}=(2 n+1)^{2} \frac{\pi m}{\hbar T}=\Delta \frac{m(2 n+1)^{2}}{2 \hbar^{2}} .
$$

Using expression (4.12) for the symmetric case and expression (4.16) we find the dependence of transmission coefficient $D$ through barrier on the oscillation period $T$ and on the value of $\Delta$ : 


$$
\begin{aligned}
& D_{n}(T)=\left\{1+\frac{\sinh ^{2}\left(2 a \sqrt{\frac{2 m U_{0}}{\hbar^{2}}-(2 n+1)^{2} \frac{\pi m}{\hbar T}}\right)}{4(2 n+1)^{2} \frac{\pi m}{\hbar T}\left(1-(2 n+1)^{2} \frac{\pi \hbar}{2 U_{0} T}\right)}\right\}^{-1} . \\
& D_{n}(\Delta)=\left\{1+\frac{\sinh ^{2}\left(2 a \sqrt{\frac{2 m U_{0}}{\hbar^{2}}-\Delta \frac{(2 n+1)^{2}}{2 \hbar^{2}}}\right)}{\Delta \frac{2 m(2 n+1)^{2}}{\hbar^{2}}\left(1-\Delta \frac{(2 n+1)^{2}}{4 U_{0}}\right)}\right\}^{-1} .
\end{aligned}
$$

Also note, that in both limiting cases $D \rightarrow 0$ and $D \rightarrow \infty$ the oscillation period $T$ approaches the value:

$$
T=\frac{4 m(b-a)^{2}}{\pi \hbar}
$$

\section{DOUBLE-WELL MORSE'S POTENTIAL}

Consider a system the potential of which is shown in Fig. 2 and is given by

$$
U(x)= \begin{cases}A\left(e^{-2 \alpha(x+c)}-2 e^{-\alpha(x+c)}\right), & \text { for }-d<x<0, \\ B\left(e^{2 \beta(x-a)}-2 e^{\beta(x-a)}\right), & \text { for } 0<x<b .\end{cases}
$$

For this potential we find solutions of stationary Schrödinger equation. Performing the following changes:

$$
\begin{array}{ll}
\xi_{A}=\frac{2 \sqrt{2 m A}}{\alpha \hbar} e^{-\alpha(x+c)}, & \text { for }-d<x<0, \\
\xi_{B}=\frac{2 \sqrt{2 m B}}{\beta \hbar} e^{\beta(x-a)}, & \text { for } 0<x<b,
\end{array}
$$

and introducing the following notations:

$$
\begin{aligned}
& s_{A}=\frac{\sqrt{-2 m E}}{\alpha \hbar}, n_{A}=\frac{\sqrt{2 m A}}{\alpha \hbar}-\left(s_{A}+\frac{1}{2}\right), \\
& s_{B}=\frac{\sqrt{-2 m E}}{\beta \hbar}, n_{B}=\frac{\sqrt{2 m B}}{\beta \hbar}-\left(s_{B}+\frac{1}{2}\right),
\end{aligned}
$$

we transform the stationary Schrödinger equation to the following form:

$\left.\left\{\frac{d^{2} \varphi}{d \xi^{2}}+\frac{1}{\xi} \frac{d \varphi}{d \xi}+\left(-\frac{1}{4}+\frac{n+s+1 / 2}{\xi}-\frac{s^{2}}{\xi^{2}}\right) \varphi\right\}\right|_{A, B}=0$
Here, the indexes $A$ or $B$ are used by the values $\varphi, \xi$, $n$ and $s$ with the proviso that equation (5.4) is solved in the region of left or right well, respectively. In order to obtain the general solution let us omit these indexes. Using the following changes:

$$
\begin{aligned}
& y(\xi)=\xi^{-\frac{c}{2}+\frac{1}{2}} e^{\frac{\xi}{2}} \varphi(\xi), \\
& c=1+2 s,
\end{aligned}
$$

equation (5.4) can be transformed to the confluent hypergeometric equation as follows

$$
\xi \frac{d^{2} y}{d \xi^{2}}+(c-\xi) \frac{d y}{d \xi}+n y=0 .
$$

Particular solutions of this equation can be written using the confluent hypergeometric function $F(-n, c ; \xi)$ $[9,10]$ :

$$
\begin{aligned}
& y_{1}(\xi)=F(-n, c ; \xi), \\
& y_{2}(\xi)=\xi^{1-c} F(-n-c+1,2-c ; \xi), \\
& y_{3}(\xi)=e^{\xi} F(c+n, c ;-\xi), \\
& y_{4}(\xi)=\xi^{1-c} e^{\xi} F(1+n, 2-c ;-\xi) .
\end{aligned}
$$

Consider the case $c \notin Z$ (i.e. $2 s \notin Z$ ). Then both the particular solutions $y_{3}$ and $y_{4}$ are linear dependent on $y_{1}$ and $y_{2}$, because they are transformed to $y_{1}$ and $y_{2}$ by Kummer's transformation $[9,10]$ :

$$
F(a, c ; \xi)=e^{\xi} F(c-a, c ;-\xi) .
$$


Solutions $y_{1}$ and $y_{2}$ are linearly independent between themselves. It can see this from its behaviour at $\xi \rightarrow 0$. Then the general solution of equation (5.6) can be written as follows

$$
\begin{aligned}
y(\xi) & =c_{1} F(-n, c ; \xi) \\
& +c_{2} \xi^{1-c} F(-n-c+1,2-c ; \xi)
\end{aligned}
$$

where $c_{1}$ and $c_{2}$ are arbitrary constants. In the initial variables the general solution has the form:

$$
\begin{aligned}
\varphi(\xi) & =e^{-\frac{\xi}{2}}\left[c_{1} \xi^{s} F(-n, 1+2 s ; \xi)\right. \\
& \left.+c_{2} \xi^{-s} F(-n-2 s, 1-2 s ; \xi)\right]
\end{aligned}
$$

Expression (5.10) with relations (5.2) and (5.3) is a general solution of the stationary Schrödinger equation at $2 s \notin Z$ and $E<0$ for the regions of left and right wells. Coefficients $c_{1 A}, c_{1 B}, c_{2 A}$ and $c_{2 B}$ (where indexes $A$ or $B$ for coefficients $c_{1}$ and $c_{2}$ are indicated at left or right well, respectively) are obtained from boundary conditions and normalization condition. Imposed boundary conditions determine the energy spectra as a discrete ones. Using them one can find the energy eigenvalues of the system.

The discrete energy spectrum determines the requirement that the wave function will be finited all over the region of its determination. The finity of the wave function in the regions $-d<x<0$ and $0<x<b$ depends on the constraining condition of particular solutions, by use of which the general solution (5.10) can be represented. Constraining particular solutions at finite values $x$ depends on constraining functions $F(-n, 1+2 s ; \xi)$ and $F(-n-2 s, 1-2 s ; \xi)$. But these functions are confluent hypergeometric and are represented by converging series at finite $\xi[10,11]$. At $n=0$ or $n \in N$ the first func- tion has the form of the polynomial, and therefore, it is bounded (because the values of $\xi$ are finited). In this fashion, at $n+2 s=0$ or $n+2 s \in N$ the second function is bounded. Therefore, both the particular solutions are bounded all over the region $-d<x<b$ (factor $\xi^{-s}$ at $\xi \rightarrow 0$ is finited because of constraining condition at $x=0$ ). Therefore, the general solution (5.10) is also bounded all over the region $-\infty<x<\infty$.

Since wave function satisfies the condition of constraining definition $x$ all over the region it can be normalized by

$$
\int_{-d}^{b}|\varphi(x)|^{2} d x=1 .
$$

Now we find the energy eigenvalues of the system. Considering possible solutions we select two cases:

1. The particle oscillates between wells along one energy level (without transitions between energy levels). In this case the energy levels which are obtained from the eigenvalue equation in the region of the left well must correspond to the energy levels which are obtained from the eigenvalue equation in the region of the right well. On this case the following boundary conditions are required:

$$
\begin{aligned}
& \varphi_{A}(0)=\varphi_{B}(0), \\
& \varphi_{A}^{\prime}(0)=\varphi_{B}^{\prime}(0), \\
& \varphi_{A}(-d)=0, \\
& \varphi_{B}(b)=0,
\end{aligned}
$$

where $\varphi_{A}(x)$ and $\varphi_{B}(x)$ are general solutions (5.10) of the stationary Schrödinger equation in the regions of left and right wells, respectively. The solution of equation set (5.12) gives the eigenvalue equation form of:

$$
\begin{aligned}
& -\frac{\alpha \xi_{A 0}}{\beta \xi_{B 0}}\left[\frac{c_{1 B}}{c_{2 B}} * \xi_{B 0}^{s_{B}} F\left(-n_{B}, 1+2 s_{B} ; \xi_{B 0}\right)+\xi_{B 0}^{-s_{B}} F\left(-n_{B}-2 s_{B}, 1-2 s_{B} ; \xi_{B 0}\right)\right] \\
& \times\left\{\frac { c _ { 1 A } } { c _ { 2 A } } * \left[-\frac{1}{2} \xi_{A 0}^{s_{A}} F\left(-n_{A}, 1+2 s_{A} ; \xi_{A 0}\right)+s_{A} * \xi_{A 0}^{s_{A}-1} F\left(-n_{A}, 1+2 s_{A} ; \xi_{A 0}\right)\right.\right. \\
& \left.+\xi_{A 0}^{s_{A}} \frac{\partial F\left(-n_{A}, 1+2 s_{A} ; \xi_{A 0}\right)}{\partial \xi_{A}}\right]+\left[-\frac{1}{2} \xi_{A 0}^{-s_{A}} F\left(-n_{A}-2 s_{A}, 1-2 s_{A} ; \xi_{A 0}\right)\right. \\
& \left.\left.-s_{A} * \xi_{A 0}^{-s_{A}-1} F\left(-n_{A}-2 s_{A}, 1-2 s_{A} ; \xi_{A 0}\right)+\xi_{A 0}^{-s_{A}} \frac{\partial F\left(-n_{A}-2 s_{A}, 1-2 s_{A} ; \xi_{A 0}\right)}{\partial \xi_{A}}\right]\right\} \\
& =\left\{\frac { c _ { 1 B } } { c _ { 2 B } } \left[-\frac{1}{2} \xi_{B 0}^{s_{B}} F\left(-n_{B}, 1+2 s_{B} ; \xi_{B 0}\right)+s_{B} * \xi_{B 0}^{s_{B}-1} F\left(-n_{B}, 1+2 s_{B} ; \xi_{B 0}\right)\right.\right. \\
& \left.+\xi_{B 0}^{s_{B}} \frac{\partial F\left(-n_{B}, 1+2 s_{B} ; \xi_{B 0}\right)}{\partial \xi_{B}}\right]+\left[-\frac{1}{2} \xi_{B 0}^{-s_{B}} F\left(-n_{B}-2 s_{B}, 1-2 s_{B} ; \xi_{B 0}\right)\right.
\end{aligned}
$$




$$
\begin{aligned}
& \left.\left.-s_{B} * \xi_{B 0}^{-s_{B}-1} F\left(-n_{B}-2 s_{B}, 1-2 s_{B} ; \xi_{B 0}\right)+\xi_{B 0}^{-s_{B}} \frac{\partial F\left(-n_{B}-2 s_{B}, 1-2 s_{B} ; \xi_{B 0}\right)}{\partial \xi_{B}}\right]\right\} \\
& \times\left[\frac{c_{1 A}}{c_{2 A}} * \xi_{A 0}^{s_{A}} F\left(-n_{A}, 1+2 s_{A} ; \xi_{A 0}\right)+\xi_{A 0}^{-s_{A}} F\left(-n_{A}-2 s_{A}, 1-2 s_{A} ; \xi_{A 0}\right)\right]
\end{aligned}
$$

where

$$
\begin{gathered}
\frac{c_{1 A}}{c_{2 A}}=-\left(\xi_{A 0} e^{\alpha d}\right)^{-2 s_{A}} \frac{F\left(-n_{A}-2 s_{A}, 1-2 s_{A} ; \xi_{A 0} e^{\alpha d}\right)}{F\left(-n_{A}, 1+2 s_{A} ; \xi_{A 0} e^{\alpha d}\right)} \\
\frac{c_{1 B}}{c_{2 B}}=-\left(\xi_{B 0} e^{\beta b}\right)^{-2 s_{B}} \frac{F\left(-n_{B}-2 s_{B}, 1-2 s_{B} ; \xi_{B 0} e^{\beta b}\right)}{F\left(-n_{B}, 1+2 s_{B} ; \xi_{B 0} e^{\beta b}\right)} \\
\xi_{A 0}=\frac{2 \sqrt{2 m A}}{\alpha \hbar} e^{-\alpha c} \\
\xi_{B 0}=\frac{2 \sqrt{2 m B}}{\beta \hbar} e^{-\beta a} .
\end{gathered}
$$

We shall transform expressions (5.3) to form:

$$
\begin{aligned}
& s_{B}=\frac{\alpha}{\beta} s_{A}, \\
& n_{B}=\frac{\alpha}{\beta} \sqrt{\frac{B}{A}}\left(n_{A}+s_{A}+\frac{1}{2}\right)-\left(\frac{\alpha}{\beta} s_{A}+\frac{1}{2}\right) .
\end{aligned}
$$

To find the eigenvalues we need to substitute expressions (5.14) and (5.16) into equation (5.13) and to resolve it relatively for the value $s_{A}$ which unequivocally defines eigenvalue $E$. The solution of equation (5.13) is performed by the use of numerical methods and determines the energy eigenvalues corresponding to the particle oscillations between wells.

2. Now we shall consider a different case: the particle oscillates in one well (for example, in the left one). In this case its full reflection takes place from the middle of the barrier (here, the transition of the particle to another energy level is possible, which exists in both regions, with further tunneling of the particle along it). Note, that in this case the reflection of the particle from the barrier is principally possible along the energy levels of the range of $0>E>U_{0}$ (this case of reflection is impossible for symmetric potential). We determine the condition of the particle reflection from the barrier as follows

$$
\varphi_{A}(0)=0 .
$$

Using this condition and also the following boundary condition

$$
\varphi_{A}(-d)=0 .
$$

we obtain equation from which the energy eigenvalues for the particle oscillations in the left well can be found

$$
\begin{aligned}
& e^{2 s_{A} \alpha d} F\left(-n_{A}-2 s_{A}, 1-2 s_{A} ; \xi_{A 0}\right) F\left(-n_{A}, 1+2 s_{A} ; \xi_{A 0} e^{\alpha d}\right) \\
& =F\left(-n_{A}-2 s_{A}, 1-2 s_{A} ; \xi_{A 0} e^{\alpha d}\right) F\left(-n_{A}, 1+2 s_{A} ; \xi_{A 0}\right)
\end{aligned}
$$

Resolving in this equation the relatively unknown values of $s_{A}$ we can find the energy eigenvalues of the system. In this fashion, the equation determining the energy eigenvalues for the particle oscillations in the right well can be obtained 


$$
\begin{aligned}
& e^{2 s_{B} \beta b} F\left(-n_{B}-2 s_{B}, 1-2 s_{B} ; \xi_{B 0}\right) F\left(-n_{B}, 1+2 s_{B} ; \xi_{B 0} e^{\beta b}\right) \\
& =F\left(-n_{B}-2 s_{B}, 1-2 s_{B} ; \xi_{B 0} e^{\beta b}\right) F\left(-n_{B}, 1+2 s_{B} ; \xi_{B 0}\right)
\end{aligned}
$$

Equations (5.19) and (5.20) include the case when some energy levels of left well are equal to some energy levels of the right well. In this case the following condition is fulfilled:

$$
\begin{aligned}
& \varphi_{A}(0)=\varphi_{B}(0)=0, \\
& \varphi_{A}^{\prime}(0)=\varphi_{B}^{\prime}(0)=0,
\end{aligned}
$$

which correspond to the particle oscillations between the wells. Therefore, it is necessary to except such energy levels from the analysis of the particle behaviour in one well.

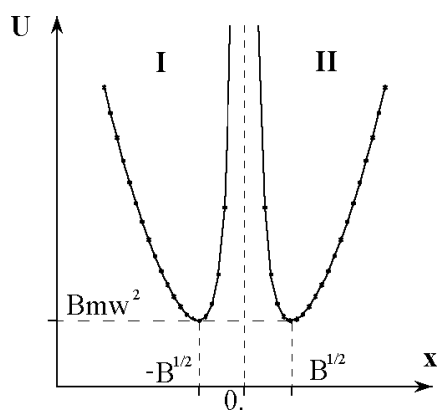

Fig. 5. Symmetric potential of form $x^{2}+B^{2} / x^{2}$.

Let us consider symmetric potential. In this case every energy level obtained by the solution of the eigenvalue equation in the left well region, is equal to the corresponding energy level which is obtained by solving the eigenvalue equation in the region of the right well. Therefore, the particle located on any energy level of the region of $E<0$ will be oscillated between the wells. The wave function becomes symmetric or antisymmetric. A system of equations for finding energy eigenvalues is given by

$$
\varphi(-d)=0, \varphi(0)=0
$$

if wave function is symmetric (even states), and

$$
\varphi(-d)=0, \varphi^{\prime}(0)=0
$$

if wave function is antisymmetric (odd states).

\section{SYMMETRIC POTENTIAL OF THE FORM OF $x^{2}+B^{2} / x^{2}$}

To analyze the particle behavior in a symmetric potential with sufficiently high barrier, we can use the potential of the following form:

$$
U(x)=\frac{m w^{2}}{2}\left(x^{2}+\frac{B^{2}}{x^{2}}\right)
$$

where $x \in]-\infty ;+\infty[$. This potential is shown on Fig. 5 . Let us use the new parameters:

$$
\begin{aligned}
& G=\frac{2 m E}{\hbar^{2}}, \\
& F=-\frac{m^{2} w^{2}}{\hbar^{2}}, \\
& K=-\frac{m^{2} w^{2}}{\hbar^{2}} B^{2} .
\end{aligned}
$$

Then the stationary Schrödinger equation is transformed to the form of

$$
\frac{d^{2} \varphi}{d x^{2}}+\left(G+F x^{2}+\frac{K}{x^{2}}\right) * \varphi=0
$$

Let us find solutions of this equation. We shall perform the following change of variables:

$$
\begin{aligned}
& \xi=\alpha x^{2}, \\
& \alpha=\frac{m w}{\hbar}=\sqrt{-F}, \\
& \varphi(\xi)=(\xi / \alpha)^{-1 / 4} w(\xi) .
\end{aligned}
$$

As a result, equation (6.3) is transformed to the standard Whittaker's form $[9,10]$ :

$$
\frac{d^{2} w}{d \xi^{2}}+\left[-\frac{1}{4}+\frac{G}{4 \sqrt{-F}} \frac{1}{\xi}+\left(\frac{3}{16}+\frac{K}{4}\right) \frac{1}{\xi^{2}}\right] w=0
$$

Using the following parameters and performing the following change of variables:

$$
k=\frac{G}{4 \sqrt{-F}},
$$




\section{S. OLKHOVSKY, S. P. MAIDANYUK}

$$
\begin{gathered}
\mu^{2}=\frac{1}{16}-\frac{K}{4}, \\
a=\frac{1}{2}-k+\mu, \\
c=1+2 \mu, \\
y(\xi)=\xi^{-c / 2} e^{\xi / 2} w(\xi),
\end{gathered}
$$

we transform equation (6.5) to a confluent hypergeometric equation of the form:

$$
\xi \frac{d^{2} y}{d \xi^{2}}+(c-\xi) \frac{d y}{d \xi}-a y=0
$$

Particular solutions of this equation can be represented by confluent hypergeometric function $F(a, c ; \xi)$ as follows

$$
\begin{aligned}
& y_{1}(\xi)=F(a, c ; \xi), \\
& y_{2}(\xi)=\xi^{1-c} F(a-c+1,2-c ; \xi), \\
& y_{3}(\xi)=e^{\xi} F(c-a, c ;-\xi), \\
& y_{4}(\xi)=\xi^{1-c} e^{\xi} F(1-a, 2-c ;-\xi) .
\end{aligned}
$$

Let us consider the case $c \notin Z$. In accordance with Kummer's transformation $[9,10]$, the solutions $y_{3}$ and $y_{4}$ can be written through $y_{1}$ and $y_{2}$. Therefore, the solutions $y_{3}$ and $y_{4}$ are linear dependent on $y_{1}$ and $y_{2}$. Write the first two solutions $y_{1}$ and $y_{2}$ in the initial variables:

$$
\begin{aligned}
& \varphi_{1}(x)=\alpha^{1 / 2+\mu} x^{-1+2 \mu} e^{-\alpha x^{2} / 2} F\left(a, 1+2 \mu ; \alpha x^{2}\right), \\
& \varphi_{2}(x)=\alpha^{1 / 2+\mu} x^{-1} e^{-\alpha x^{2} / 2} F\left(a-2 \mu, 1-2 \mu ; \alpha x^{2}\right) .
\end{aligned}
$$

The condition of the wave function finity all over the range $x$ requires, that the following conditions are fulfilled:

$$
\begin{aligned}
& \text { for } \varphi_{1}(x): a \in 0,-N ; 2 \mu \notin-N, \\
& \text { for } \varphi_{2}(x):-a+2 \mu \in 0, N ; 2 \mu \notin N .
\end{aligned}
$$

As a result of these conditions, the series, which represent a confluent hypergeometric function for the solutions $\varphi_{1}$ and $\varphi_{2}$, transform to polynomial and the spectrum becomes discrete. The analysis of expressions (6.11) shows that solutions $\varphi_{1}$ and $\varphi_{2}$ cannot be used at the same time. But both the solutions have equal energy eigenvalues $E$ which can be written as

$$
E_{n}^{ \pm}=2 \hbar w\left(\frac{1}{2}+n \pm \frac{1}{4} \sqrt{1+\frac{4 m^{2} w^{2}}{\hbar^{2}} B^{2}}\right)
$$

where $n \in 0, N$. The general solution of the wave function can be represented through $\varphi_{1}$ or $\varphi_{2}$ (which are linearly independed between themselves). Both the energy eigenvalues $E_{n}^{+}$and $E_{n}^{-}$used in expression (6.12) can be considered separately as defining two independed waves with the oscillation periods $T^{+}$and $T^{-}$, respectively. To obtain a period for every wave we write equation (6.12) as follows

$$
\begin{aligned}
E_{n}^{ \pm} & =E_{0}^{ \pm}+\Delta n \\
& =2 \hbar w n+2 \hbar w\left(\frac{1}{2} \pm \frac{1}{4} \sqrt{1+\frac{4 m^{2} w^{2}}{\hbar 2} B^{2}}\right)
\end{aligned}
$$

From this we obtain

$$
\begin{aligned}
& E_{0}^{ \pm}=2 \hbar w\left(\frac{1}{2} \pm \frac{1}{4} \sqrt{1+\frac{4 m^{2} w^{2}}{\hbar 2} B^{2}}\right), \\
& \Delta=2 \hbar w \\
& T^{ \pm}=\frac{2 \pi \hbar}{\Delta}=\frac{\pi}{w} .
\end{aligned}
$$

It can be seen from expressions (6.13), that the two considered waves have equal periods. In accordance with the following relation

$$
A e^{i w t+i \delta_{1}}+B e^{i w t+i \delta 2}=e^{i w t}\left(A e^{i \delta_{1}}+B e^{i \delta_{2}}\right),
$$

it can be seen that the general period of oscillations between the wells is equal to $T^{+}$or $T^{-}$:

$$
T=T^{ \pm}=\frac{\pi}{w} .
$$

Now we find a dependence of the transmission coefficient $D$ through the barrier on the oscillation period $T$ between the wells. Consider the case of small values of $D$ when expression (3.4) can be used. From expression (6.13) we find the distance between two closely located levels by

$$
\Delta E=E_{n}^{+}-E_{n}^{-}=\hbar w \sqrt{1+\frac{4 m^{2} w 2}{\hbar^{2}} B^{2}}
$$

Using this relation and also expression (3.4), we obtain the transmission coefficient as follows

$$
D \sim \pi^{2}\left(1+\frac{4 m^{2} w^{2}}{\hbar^{2}} B^{2}\right)=\pi^{2}\left(1+\frac{m^{2} B^{2}}{\hbar^{4}} \Delta^{2}\right)
$$




$$
=\pi^{2}\left(1+\frac{\pi^{2} 16 m^{2} B^{2}}{\hbar^{2}} \frac{1}{T^{2}}\right)
$$

This expression establishes the dependencies of the transmission coefficient $D$ on the oscillation period $T$ between the wells and on the largest common divizor $\Delta$ which is determined by expression (1.1).

\section{DOUBLE-WELL SYMMETRIC PARABOLIC POTENTIAL}

Consider the system the potential of which can be written as

$$
U(x)=\left\{\begin{array}{l}
\frac{1}{2} m w^{2}(x+a)^{2}, \text { for } x<0 \\
\frac{1}{2} m w^{2}(x-a)^{2}, \text { for } x>0
\end{array}\right.
$$

Let us assume that potential $U(x)$ satisfies conditions of using the WKB approximation. As a result of tunneling through a barrier the displacements of energy levels which takes place because of level splitting expression (3.1) is determined from their positions without tunneling. For potential (7.1) the energy eigenvalues are given by

$$
\begin{aligned}
E_{n}^{-} & =\hbar w\left(n+\frac{1}{2}\right)-\Delta E_{n}, \\
E_{n}^{+} & =\hbar w\left(n+\frac{1}{2}\right)+\Delta E_{n}, \\
\Delta E_{n} & =\left|E_{n}^{ \pm}-E_{n}^{(0)}\right|=\frac{\hbar^{2}}{m} \varphi_{n}^{0}(0) \frac{\partial \varphi_{n}^{(0)}(0)}{\partial x}
\end{aligned}
$$

where $E_{n}^{-}$and $E_{n}^{+}$are eigenvalues occuring because of splitting and corresponding to symmetric and antisymmetric wave functions, respectively. $\varphi_{n}^{(0)}$ is a solution of stationary Schrödinger equation in the region $x>0$ without splitting and has the form:

$$
\varphi_{n}^{(0)}(x)=A_{n} e^{-\alpha^{2}(x-a)^{2} / 2} H_{n}(\alpha(x-a))
$$

where $\alpha=\sqrt{m w / \hbar}, H_{n}(\xi)$ is the Hermitian polynomial. Using (7.3), we find the displacement value:

$$
\Delta E_{n}=\frac{\hbar^{2}}{m} A_{n}^{2} e^{-\alpha^{2} a^{2}} H_{n}(\alpha a)\left(\alpha^{2} a H_{n}(\alpha a)-2 n H_{n-1}(\alpha a)\right)
$$

Coefficient $A_{n}$ can be obtained from the normalization condition of $\varphi_{n}^{(0)}(x)$. The function $\varphi_{n}^{(0)}(x)$ is assumed as a normalized one whose integral of $\left|\varphi_{n}^{(0)}\right|^{2}$ in the region of the right well is equal to 1 . Then we can write

$$
\begin{aligned}
A_{n}^{2} & =\left\{\int_{0}^{+\infty} e^{-\alpha^{2}(x-a)^{2}} H_{n}^{2}(\alpha(x-a)) d x\right\}^{-1} \\
& =\frac{\alpha}{2^{n} n !}\left\{\frac{\sqrt{\pi}}{2}(1+\operatorname{erf}(\alpha a))\right. \\
& \left.-e^{-\alpha^{2} a^{2}} \sum_{k=0}^{n-1} \frac{H_{n-k}(\alpha a) H_{n-k-1}(\alpha a)}{(n-k) !}\right\}^{-1}
\end{aligned}
$$

where $\operatorname{erf}(x)=\frac{2}{\sqrt{\pi}} \int_{0}^{x} e^{-\xi^{2}} d \xi$ is the integral of probability [10].

\section{CONCLUSIONS}

In all the problems considered above an attempt to describe the tunneling process of the particle through the barrier with its oscillations between the wells in double-well symmetric potential was made on the basis of such main parameters as period $T$ of the particle oscillations between the wells, the transmission coefficient $D$ through a barrier, reflection coefficient $R$ from the barrier (for squared potential). The transmission coefficient $D$ through the barrier is found with consideration of the particle which is incident upon the barrier having asymptotic velocity $\hbar k / m$ and is initially determined for continuous energy spectrum (see $[2,4]$ ). One can obtain all the considered above parameters and describe the particle tunneling through a barrier from the found energy eigenvalues of the considered systems. If the transmission coefficient is small, then it can be calculated in the WKB approximation for a discrete energy spectrum. Comparison of both the methods realized for a squared potential shows that the values of transmission coefficient calculated by these methods are equal with the accuracy to normalization constant which is determined by accuracy of the used WKB approximation and equal to 1 for the first order terms in $\hbar$. Therefore, the transmission coefficient of the particle through a barrier between two wells in a double-well potential is considered only formally, if it was initially determined for the continuous spectrum, or with the accuracy to the normalization constant, if it is obtained in the WKB approximation.

The dependencies of transmission coefficient when its value is small on another parameters are identical for both the methods. These dependencies are obtained, if the parameters are the oscillation period between wells and the largest common divizor determined by expression (1.1) when the WKB approximation is applied.

All the parameters considered above and describing the tunneling of the particle through a barrier can be obtained on the basis of the discovered energy levels of the systems considered. For asymmetric forms of the potential they are more difficult to found. 


\title{
V. S. OLKHOVSKY, S. P. MAIDANYUK
}

For asymmetric forms of potentials the splitting of energy levels which occurs because of tunneling of the particle through a barrier and is studied by the theory of WKB approximation gives some features which are absent when the potential is symmetric (for example, the possibility of the particle reflection from a barrier along the level located higher than the height of a barrier). In the case of the potential asymmetry the use of boundary conditions must be careful enough. Therefore, in the present work a considerable attention is paid to the problem of finding the solutions of energy levels for asymmetric potentials.

The problem of the squared potential was studied early in literature (for example, see [2-7]). This problem is considered here as one of the simplest cases because it can give a visual teaching picture for studying the tunneling particle behavior. Relative easiness of finding the solutions in comparison with the problems with rounded off potentials allows to study deeply the process of tunneling on the basis of oscillation period and transmission and reflection coefficients.

It is significantly difficult to obtain solutions for the potential which has a rounded off form. Using doublewell Morse's potential (as an example) the solution existence is studied and energy eigenvalues equation is obtained a further solution of which can be calculated by the use of numerical methods.

The interesting symmetric double-well potential of the form $x^{2}+B^{2} / x^{2}$ is also analyzed because the exact analytical solutions exist for it (and one can obtain energy eigenvalues $E_{n}$, period $T$ of oscillations between wells in the explicit form). This potential is suitable enough for the analysis of the particle behavior in wells with a sufficiently high barrier (with sufficiently large depth). Models of a one-dimensional motion of the particle in such a form of potential have been studied in the literature [8].

Further, basic parameters the determining behavior of the particle motion in some forms of asymmetric doublewell potentials are to be obtained.
[1] V. S. Olkhovsky, V. S. Savoshchenko, Izv. Vyssh. Uchebn. Zaved., Fiz. 10, 58 (1989).

[2] L. D. Landau, E. M. Lifshits, Kvantovaia miekhanika (Quantum Mechanics) (Nauka, Moscow, 1989).

[3] L. Schiff, Kvantovaia miekhanika (Quantum Mechanics) (IL, Moskow, 1957).

[4] M. Razavy, Ashok Pimpale, Phys. Rep. 168, 305 (1988).

[5] A. Anderson, Am. J. Phys. 57, 230 (1989).

[6] P. Senn, Am. J. Phys. 60, 228 (1992).

[7] W. D. Heiss, W. H. Steeb, Phys. Rev. A 39, 6018 (1989).

[8] V. S. Otchik, Vestsi Akad. Navuk Belarusi, Ser. Fiz.-
Mat. Navuk 4, 66 (1996).

[9] H. Bateman, A. Erdélyi, Vysshyie transtsendientnyie funktsii, t. 1 (Higher Transcendential Functions) (Nauka, Moscow, 1973).

[10] Spravochnik po spietsial'nym funktsiiam s formulami, grafikami i matiematicheskimi tablitsami (Handbook of Mathematical Functions with Formulas, Graphs and Mathematical Tables), edited by M. Abramovitz, I. Stegun (Nauka, Moscow, 1979).

[11] N. N. Vorobiov, Tieoriia riadov (Theory of Series) (Nauka, Moscow, 1986).

\section{ПРО ЕВОЛЮЦІЮ ПЕРЕХОДІВ 3 ОДНІЕЇ ЯМИ В ІНШУ У ДВОЯМНОМУ ПОТЕНЦІяЛ І}

\author{
В. С. Ольховський, С. П. Майданюк \\ Інститут ядерних досліджень НАН Украйни, \\ просп. Науки, 47, 252650, Киїь-22, Украӥна \\ E-mail:maidan@kinr.kiev.ua
}

\begin{abstract}
Розглянуто одновимірну за дачу з різними потенціялами, які мають вигляд двох ям, розділених бар'єром. Вивчаємо можливість тунелювання крізь бар'єр частинки, яка спочатку знаходиться в одній із ям, а потім осцилює між ними. Для асиметричного прямокутного потенціялу та асиметричного потенціялу Морзе отримані рівняння для знаходження власних значень рівнів енергії, подальший розв'язок яких може бути виконано чисельно. Для прямокутного потенціялу при його симетрії отримано зале жність періоду осциляцій частинки між ямами, який визначається на основі знайдених рівнів енергї̈ в першому набли женні, від коефіцієнта проходження частинки крізь бар'єр.

Коефіцієнт проходження може бути отриманий двома способами: для частинки в області неперервного спектра енергій, яка спочатку має асимптотичну пвидкість $\hbar k / m$, а потім падаєна бар'єр, та для частинки в області дискретного спектра, коли може бути використано ВКБ-наближення. Обидва підходи дають вирази для коефіцієнта проходження, які збігаються з точністю до константи, що визначається точністю використання ВКБ-наближення.
\end{abstract}

Для потенщіялу виду $x^{2}+B^{2} / x^{2}$ отримані точні аналітичні розв'язки для рівнів енергї та періоду осциляціӥ. 